\title{
Evaluation of Garlic Extract Gel as Local Drug Delivery in the Treatment of Chronic Periodontitis: A Clinical Study
}

\author{
Ravi T Reddy ${ }^{1}$, Vandana KVP Kumar ${ }^{2}$, Shobha Prakash ${ }^{3}$
}

\begin{abstract}
Objective: Here an attempt is made to assess the clinical benefits of garlic extract gel when used as an adjunct to nonsurgical periodontal therapy. Materials and methods: A total of 13 patients with periodontitis (AAP criteria) were selected. A split mouth design was used and sites in each patient were allocated into the experimental group and the control group. In the experimental group, sites were treated with scaling and root planing (SRP) + garlic gel. In the control group, sites were treated with SRP alone. Plaque index (PI), gingival bleeding index (GBI), gingival index (GI), probing pocket depth (PPD), and clinical attachment level (CAL) were assessed at baseline on 21 st day, 30th day, and 90 th day.

Results: On intragroup comparison, the reduction in clinical parameters like PI, GI, GBI, and PPD was highly significant in both test and control groups ( $p \leq 0.001$ ), whereas CAL showed no significant gain in the control group but highly statistical significant result was seen in the test group. On intergroup comparison, all the clinical parameters like PI and CAL showed statistically significant $(p \leq 0.05)$ improvement the in test group when compared with that of the control group, whereas $\mathrm{Gl}, \mathrm{GBI}$, and PPD showed statistically high significant $(p \leq 0.001)$ improvement in subjects with SRP + garlic gel when compared with SRP alone.

Conclusion: These results conclude that garlic gel can be a used as an antibacterial and anti-inflammatory agent in adjunct to SRP. However, further research with larger samples needs to be focused to confirm the use of garlic in clinical practice.

Keywords: Clinical attachment level, Garlic, Herbal, Local drug delivery, Periodontitis, Probing depth, Scaling and root planing. CODS Journal of Dentistry (2018): 10.5005/jp-journals-10063-0044
\end{abstract}

\section{INTRODUCTION}

The word "periodontitis" comes from "peri" (around), "odont" (tooth), and "itis" (redness). Periodontitis is a set of diseases, which usually attacks the periodontium. Periodontitis in comparison with gingivitis is a more severe inflammation, because not only it affects the tissues but also the bottom of the teeth. If it is not treated at all, it may lead to loss of teeth., ${ }^{1,2}$

Evidence implicates dental plaque as the causative agent of gingivitis, and specific organisms including Porphyromonas gingivalis, Prevotella intermedia, Fusobacterium nucleatum, Campylobacter rectus (formerly Wolinella recta), Eikenella corrodens, and possibly spirochetes as causative agents in adult forms of periodontitis. ${ }^{3}$

The host response to antigens and irritants released by bacteria includes the local release of antibodies, lymphocytes, and neutrophil activation and their infiltration into the gingival tissue. The activation of lymphocytes and neutrophils is defensive and involves bacterial as well as possible tissue destruction.

Scaling and root planing (SRP) is a traditional method that has been shown to be an effective treatment for chronic periodontitis. Although mechanical treatment significantly decreases the prevalence and levels of subgingival microorganisms, it does not necessarily eliminate all pathogens. To overcome these problems, surgical procedure and local and systemic antibiotics have been employed to facilitate the elimination of subgingival microflora. ${ }^{4}$

However, systemic antibiotics require the administration of large dosages to obtain suitable concentrations at the site of disease, which could potentially promote the development of side effects, drug interactions, and inconsistent patient compliance. To avoid these limitations of systemic administration, a different approach has been introduced that uses local delivery systems that contain antibiotic or antiseptic agents. ${ }^{5}$
${ }^{1-3}$ Department of Periodontics, College of Dental Sciences, Davangere, Karnataka, India

Corresponding Author: Ravi T Reddy, Department of Periodontics, College of Dental Sciences, Davangere, Karnataka, India, Phone: +91 8884327217, e-mail: raviteja.palley@gmail.com

How to cite this article: Reddy RT, Kumar VKVP, Prakash S. Evaluation of Garlic Extract Gel as Local Drug Delivery in the Treatment of Chronic Periodontitis: A Clinical Study. CODS J Dent 2018;10(1):1-6.

Source of support: Nil

Conflict of interest: None

The common agents used include subgingival chlorhexidine, tetracycline fibers, subgingival minocycline, subgingival doxycycline, and subgingival metronidazole. However, prolonged use of systemic antibiotics can result in the development of resistant strains. With the rise in bacterial resistance to antibiotics, there is considerable interest in the development of other classes of antimicrobials for the control of infection. Currently, the use of herbal products in dentistry is ever increasing. This can be attributed to their easy availability, low cost, and less side effects. ${ }^{6}$

As there are approximately 500,000 plant species occurring worldwide, of which only $1 \%$ has been phytochemically investigated, there is great potential for discovering novel bioactive compounds. $^{7}$

Botanicals like Acacia Catechu, Cinnamomum zeylanicum, Allium sativum, Propolis, Mikania laevigata, Mikania glomerata, Droserapeltata, Helichrysum italicum, Coptidis rhizome, Piper cubeba, Azadirachta indica, Syzygium aromaticum, and tea tree oil (Melaleuca alternifolia) are some potential antimicrobial deriving agents used in the management of dental infections. ${ }^{8}$ 


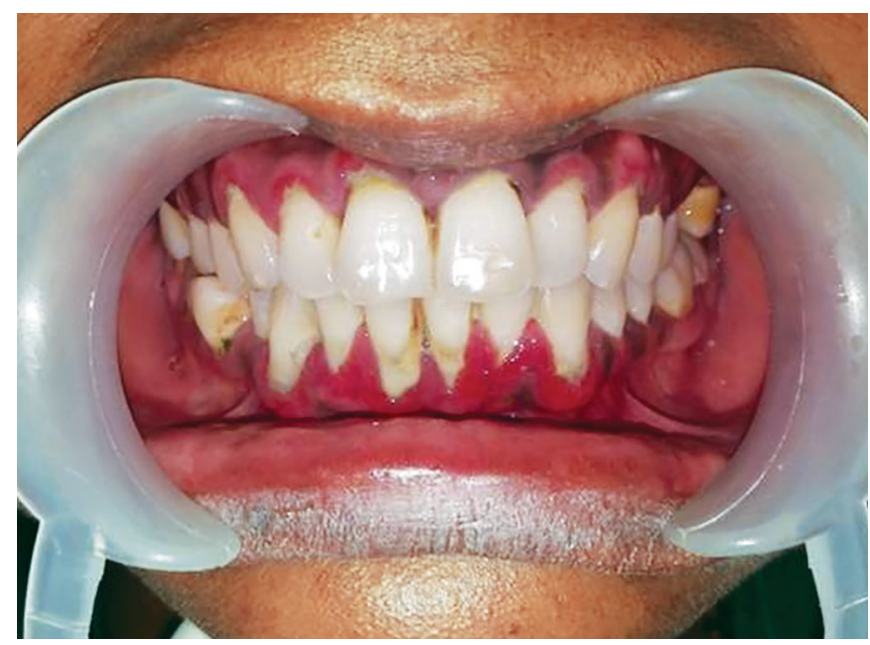

Fig. 1: Preoperative clinical picture

One such herb with wide medicinal importance is Allium sativum L., commonly called as garlic. It is known to have antimicrobial, antiinflammatory, antioxidant, and antiviral properties. ${ }^{9}$

Here an attempt is made to evaluate garlic extract gel as a local drug delivery (LDD) in the treatment of chronic periodontitis patients clinically.

\section{Materials and Methods}

Thirteen systemically healthy chronic periodontitis patients (Fig. 1) were included in this double-blind, randomized, parallel-design, clinical trial, to evaluate the effect of garlic extract gel as a local drug (Fig. 2) with and without scaling and root planing (SRP) on the clinical parameters and the subgingival microbiological profile of chronic periodontitis patients.

The subjects for this study were selected from the outpatient department of Periodontics, College of Dental Sciences, Davangere, Karnataka. The study protocol was conducted under the guidelines prescribed by Rajiv Gandhi University of Health Sciences, Bengaluru, and approved by the local Ethical committee at College Of Dental Sciences, Davangere, Karnataka, India. The duration of this study was $1 \frac{1}{2}$ years, ranging from January 2015 to June 2016 .

\section{Patient Selection Criteria Inclusion Criteria}

- Patients who were diagnosed as suffering from chronic periodontitis (AAP International Workshop for Classification of Periodontal Diseases, 1999). ${ }^{10}$

- Patients selected should have mild to moderate periodontal pockets $(5-7 \mathrm{~mm})$ clinically with radiographic evidence of bone loss. $^{10}$

\section{Exclusion Criteria}

- Patients suffering from any known systemic diseases.

- Patients who had received any antibiotic therapy in the last 6 months.

- Patients who had received any chemotherapeutic mouth rinses and oral irrigation during the past 1 month.

- Patients who had received any surgical or nonsurgical therapy, 6 months prior to the start of the study.

- Patients who were pregnant or lactating.

- Patients who were smokers/alcoholic.

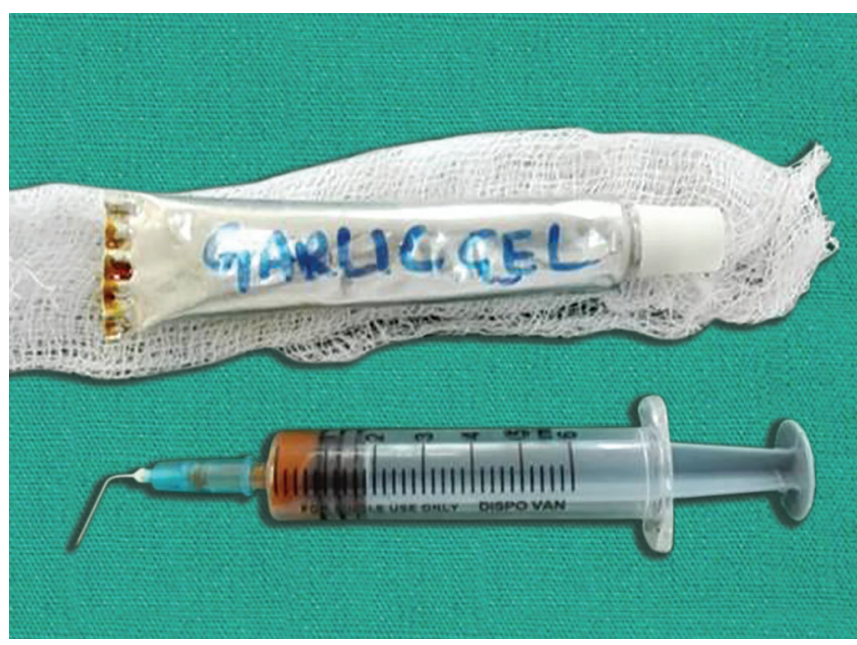

Fig. 2: Gel prepared from garlic extract

\section{Study Procedure}

The clinical parameters recorded were the following: plaque index (PI) (Silness and Loe, 1964), gingival index (GI) (Loe and Silness, 1963), gingival bleeding index (GBI) (Ainamo and Bay, 1975), probing pocket depth (PPD), and clinical attachment level (CAL).

The treatment modalities were divided according to the split mouth protocol. ${ }^{11}$ Patient's dental arch was divided into upper and lower arch. Either right or left half of the quadrant is taken as the test or control site using the coin toss method. The test site is designated as A that received SRP + garlic extract gel as LDD and the control site is designated as B where only SRP was performed. The clinical parameters were recorded at baseline, 21st day, 30th day, and 90 th day.

\section{Preparation of Garlic Gel}

The procedure was carried out at Bapuji College of pharmacy, Davangere, Karnataka, India.

\section{Extraction of Crude Drug from Garlic}

Extraction of drug is carried out using a process called maceration, where finely chopped garlic cloves that weighed $250 \mathrm{~g}$ are placed in a stoppered container with the solvent and to increase the solvent efficiency gentle heat is used and is allowed to stand for 3 days with frequent agitation until the soluble matter is dissolved; the mixture is then strained and used for preparation of gel.

\section{Preparation of Gel from the Drug \\ Preparation of $57.1 \%$ Garlic Gel \\ Preparation of Garlic Extract Gel}

Accurately weighed amount of the garlic extract is dissolved in water, then weighed amount of hydroxy propyl methyl cellulose (HPMC) is added slowly, then glycerin and cetylpyridinium chloride are added, and finally volume is made with water q.s. to $20 \mathrm{~g}$.

The concentration of the gel is set based on the previous study.

\section{Procedural Steps}

- Scaling and root planing was done for the prescribed quadrants using ultrasonic (Cavitron-BOBCAT PRO, DENTSPLY; Power-240 $A C 50 / 60 \mathrm{~Hz} 80 \mathrm{VA}$ ) and hand instruments (Universal Curettes, 2R/2L and 4R/4L Hufriedy-USA). 


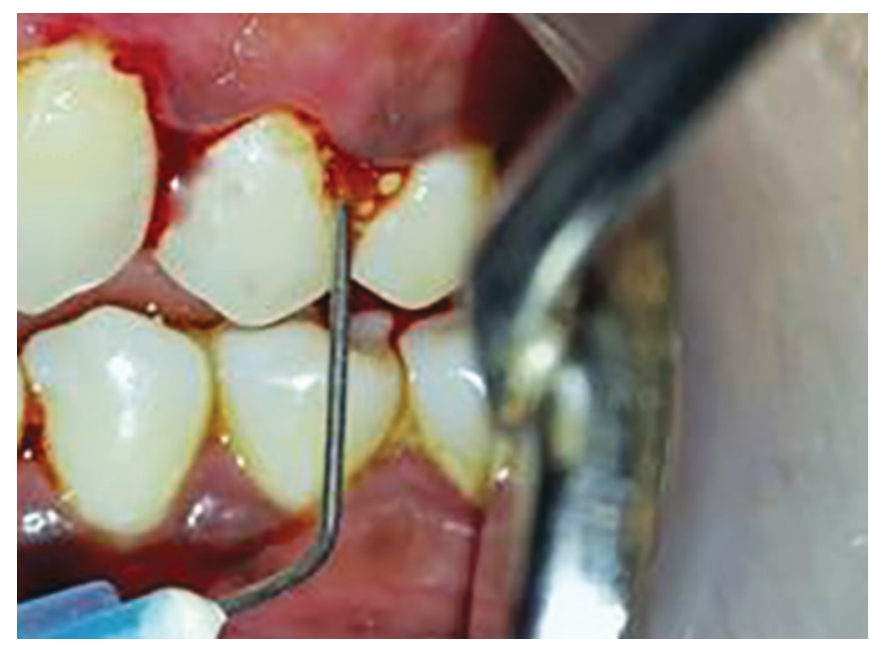

Fig. 3: Delivery of garlic gel at test sites

- Local delivery of garlic gel: the garlic extract gel was dispensed using a blunt tip. The tip was inserted till the base of the pocket and the drug was injected slowly while drawing the tip upward till the gingival margin; excess gel flowing out of the pocket was gently wiped using sterile cotton (Fig. 3).

\section{Statistical Analysis}

- Statistical analysis was done with SPSS (version 2.0) USA. Data comparison was done by applying specific statistical tests to find out the statistical significance of the results. Since the data were continuous type, parametric tests were used for analysis. Mean and standard deviation (SD) were calculated. Statistical tests employed for the obtained data in this study were the following:

- The repeated measure analysis of variance (ANOVA) test was used for intragroup comparisons at different time intervals followed by the post hoc analysis and the unpaired " $t$ " test for intergroup comparisons.

- For GBI, the parametric test using chi square was used as it was measured in percentage and was a nonparametric test.

\section{Results}

The clinical parameters at baseline (Table 1) between the experimental and control groups were statistically nonsignificant.

Intragroup comparison: (Tables 2 to 6 )

In SRP group comparison, the PI showed a mean reduction of $1.01 \pm 0.51$ between 0 day and 90th day, which was statistically highly significant $p=0.000$ ( $p \leq 0.001$ ); the $\mathrm{Gl}$ showed a mean reduction of $1.42 \pm 0.79$ between 0 day and 90th day, which was statistically highly significant $p=0.000(p \leq 0.001)$; the GBI showed a mean reduction of $66.26 \pm 2.6$ between 0 day and 90 th day, which was statistically highly significant $p=0.000$ ( $p \leq 0.001)$; PPD showed a mean reduction of $1.53 \pm 0.3$ between 0 day and 90 th day, which was statistically highly significant $p=0.000$ ( $p \leq 0.001)$; CAL showed a mean gain of $0.81 \pm 0.33$ between 0 day and 90 th day, which was not statistically significant ( $p=0.078$ ).

In SRP + garlic gel group, the PI showed a mean reduction of $1.24 \pm 0.59$ between 0 day and 90 th day, which was statistically

Table 1: Baseline results-clinical parameters

\begin{tabular}{llllll}
\hline Groups & $P I$ & $G l$ & $G B I$ & $P D$ & CAL \\
\hline SRP & $2.09 \pm 0.18$ & $1.9 \pm 0.31$ & $78.13 \pm 6.5$ & $6.8 \pm 0.83$ & $4.9 \pm 0.87$ \\
SRP + garlic gel & $2.18 \pm 0.22$ & $2.09 \pm 0.33$ & $79.11 \pm 6.87$ & $7.05 \pm 0.88$ & $5.03 \pm 0.90$ \\
$p$ value & 0.27 (NS) & 0.45 (NS) & 0.71 (NS) & 0.64 (NS) & 0.83 (NS) \\
\hline
\end{tabular}

Table 2: Intragroup and intergroup comparison: plaque index

\begin{tabular}{|c|c|c|c|c|c|c|}
\hline Group & Baseline & 21 days & 30 days & 90 days & Mean reduction & pvalue \\
\hline SRP & $2.09 \pm 0.18$ & $1.27 \pm 0.10$ & $1.22 \pm 0.09$ & $1.08 \pm 0.11$ & $1.01 \pm 0.51$ & $0.000(\mathrm{HS})$ \\
\hline SRP + garlic gel & $2.18 \pm 0.22$ & $1.16 \pm 0.10$ & $1.09 \pm 0.06$ & $0.94 \pm 0.16$ & $1.24 \pm 0.59$ & $0.000(\mathrm{HS})$ \\
\hline$p$ value & 0.27 (NS) & 0.014 & 0.00 & $0.016(\mathrm{~S})$ & & \\
\hline
\end{tabular}

$p$ value $\leq 0.05$ is significant

$p$ value $\leq 0.001$ is highly significant

Table 3: Intragroup and intergroup comparison: gingival index

\begin{tabular}{|c|c|c|c|c|c|c|}
\hline Group & Baseline & 21 days & 30 days & 90 days & Mean reduction & pvalue \\
\hline SRP & $1.9 \pm 0.31$ & $1.17 \pm 0.11$ & $0.86 \pm 0.15$ & $0.57 \pm 0.16$ & $1.42 \pm 0.79$ & $0.000(\mathrm{HS})$ \\
\hline SRP + garlic gel & $2.09 \pm 0.33$ & $0.93 \pm 0.12$ & $0.76 \pm 0.17$ & $0.31 \pm 0.12$ & $1.77 \pm 0.83$ & $0.000(\mathrm{HS})$ \\
\hline$p$ value & 0.45 (NS) & 0.000 & 0.115 & $0.000(\mathrm{HS})$ & & \\
\hline
\end{tabular}

$p$ value $\leq 0.05$ is significant

$p$ value $\leq 0.001$ is highly significant

Table 4: Intragroup and intergroup comparison: gingival bleeding index

\begin{tabular}{|c|c|c|c|c|c|c|}
\hline Group & Baseline & 21 days & 30 days & 90 days & Mean reduction & $p$ value \\
\hline SRP & $78.13 \pm 6.5$ & $40.94 \pm 9.69$ & $23.35 \pm 5.69$ & $11.86 \pm 2.60$ & $66.26 \pm 2.6$ & $0.000(\mathrm{HS})$ \\
\hline SRP + garlic gel & $79.11 \pm 6.87$ & $33.89 \pm 8.66$ & $18.69 \pm 5.29$ & $7.43 \pm 2.49$ & $71.67 \pm 2.45$ & 0.000 (HS) \\
\hline$p$ value & 0.71 (NS) & 0.06 & $0.04(\mathrm{~S})$ & $0.000(\mathrm{HS})$ & & \\
\hline
\end{tabular}

$p$ value $\leq 0.05$ is significant

$p$ value $\leq 0.001$ is highly significant 
Table 5: Intragroup and intergroup comparison: probing pocket depth

\begin{tabular}{|c|c|c|c|c|c|c|}
\hline Group & Baseline & 21 days & 30 days & 90 days & Mean reduction & $p$ value \\
\hline SRP & $6.8 \pm 0.83$ & $6.27 \pm 0.75$ & $6.13 \pm 0.77$ & $5.36 \pm 0.72$ & $1.53 \pm 0.3$ & $0.000(\mathrm{HS})$ \\
\hline SRP + garlic gel & $7.05 \pm 0.88$ & $5.36 \pm 0.7$ & $5.26 \pm 0.70$ & $4.29 \pm 0.71$ & $2.75 \pm 0.29$ & $0.000(\mathrm{HS})$ \\
\hline$p$ value & 0.64 NS & 0.04 & 0.06 & $0.001(\mathrm{HS})$ & & \\
\hline
\end{tabular}

$p$ value $\leq 0.05$ is significant

$p$ value $\leq 0.001$ is highly significant

Table 6: Intragroup and intergroup comparison: clinical attachment level

\begin{tabular}{|c|c|c|c|c|c|c|}
\hline Group & Baseline & 21 days & 30 days & 90 days & Mean reduction & $p$ value \\
\hline SRP & $4.9 \pm 0.87$ & $4.65 \pm 0.83$ & $4.55 \pm 0.83$ & $4.14 \pm 0.82$ & $0.81 \pm 0.33$ & 0.078 \\
\hline SRP + garlic gel & $5.03 \pm 0.90$ & $4.30 \pm 0.91$ & $4.21 \pm 0.90$ & $3.23 \pm 0.83$ & $1.74 \pm 0.35$ & $0.000(\mathrm{HS})$ \\
\hline$p$ value & 0.83 NS & 0.32 & 0.33 & $0.01(\mathrm{~S})$ & & \\
\hline
\end{tabular}

$p$ value $\leq 0.05$ is significant

$p$ value $\leq 0.001$ is highly significant

highly significant $p=0.000$ ( $p \leq 0.001$ ); the Gl showed a mean reduction of $1.77 \pm 0.83$ between 0 day and 90th day, which was statistically highly significant $p=0.000$ ( $p \leq 0.001)$; the GBI showed a mean reduction of $71.67 \pm 2.45$ between 0 day and 90th day, which was statistically highly significant $p=0.000$ ( $p \leq 0.001)$; PPD showed a mean reduction of $2.75 \pm 0.29$ between 0 day and 90 th day, which was statistically highly significant $p=0.000(p \leq 0.001)$; CAL showed a mean gain of $1.74 \pm 0.35$ between 0 day and 90th day, which was statistically highly significant $(p \leq 0.001)$.

\section{Intergroup Comparison}

\section{Plaque Index}

At the end of 90 days, significant reduction of PI was seen in both groups, with maximum reduction seen in the SRP + garlic gel group. Statistical significance was seen between the groups $p=0.016$ $(p \leq 0.05)$ (Table 2).

\section{Gingival Index}

At the end of 90 days, significant reduction of Gl was seen in both groups, with maximum reduction seen in the SRP + garlic gel group. High statistical significance was seen between the groups $p=0.000$ ( $p \leq 0.001$ ) (Table 3).

\section{Gingival Bleeding Index}

At the end of 90 days, significant reduction of GBI was seen in both groups, with maximum reduction seen in the SRP + garlic gel group. High statistical significance was seen between the groups $p=0.000$ ( $p \leq 0.001$ ) (Table 4).

\section{Probing Pocket Depth}

At the end of 90 days, significant reduction of PPD was seen in both groups, with maximum reduction seen in the SRP + garlic gel group. High statistical significance was seen between the groups $p=0.001$ ( $p \leq 0.001)$ (Table 5).

\section{Clinical Attachment Level}

At the end of 90 days, significant CAL gain was seen in both groups, with maximum gain seen in the SRP + garlic gel group. Statistical significance was seen between the groups $p=0.01(p \leq 0.05)$ (Table 6).

Overall, at the end of 90 days, among both the SRP group and the SRP + garlic gel group, PI showed statistically significant reduction, CAL showed statistically significant gain, and PPD, GI, and

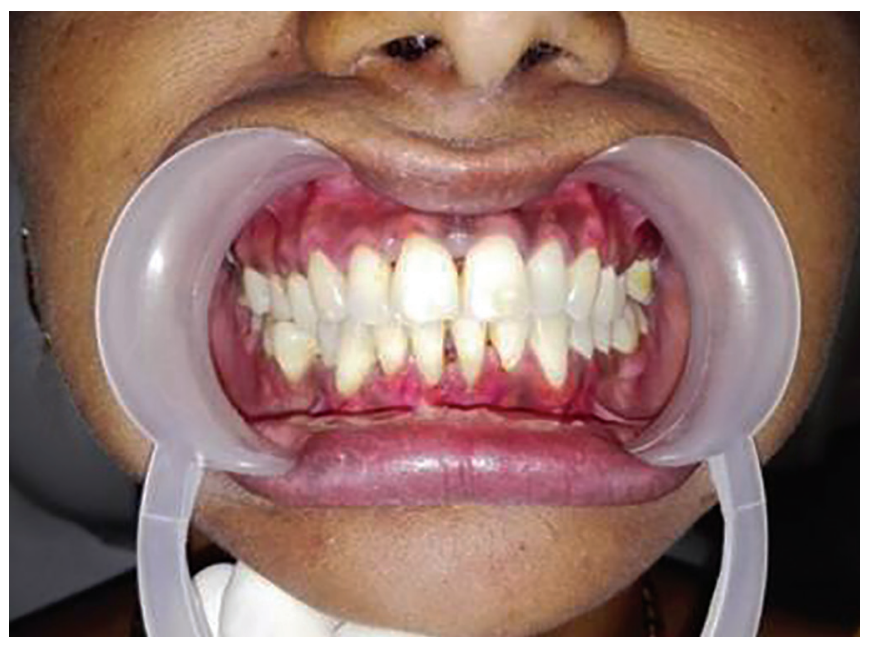

Fig. 4: Postoperative clinical picture

GBI showed highly statistical significant reduction in the SRP + garlic gel group on intergroup comparison over the SRP group (Fig. 4).

\section{Discussion}

The backbone of periodontal treatment consists of mechanical removal of bacterial deposits and calculus from the subgingival environment either by hand instruments, or ultrasonic devices, performed either surgically or nonsurgically, along with a strict regimen of plaque control, although it is known that complete removal of subgingival plaque and calculus is unrealistic, especially as pocket depth increases. ${ }^{12}$ Antibacterial agents have thus become an integral part of the therapeutic armamentarium.

Local drug delivery systems are means of drug application to confined areas. The goal is to maintain effective concentrations of chemotherapeutic agents at the site of action for long periods, despite drug loss from crevicular fluid clearance. ${ }^{13}$ Also using a controlled delivery device includes better patient compliance, better pharmacokinetic response, better access, ability to position the drug adjacent to the disease, and ability to lower total dosage of the drug at a more controlled concentration. ${ }^{14}$

A meta-analysis assessing the effect of the LDD system of chlorhexidine (CHX) as an adjunct to SRP in chronic periodontitis showed significant reduction of probing depth but no significant 
gain in the attachment level. Similarly meta-analysis of local delivery of tetracycline, metronidazole, and minocycline as an adjunct to conventional mechanical therapy suggested improved clinical outcomes. However considerations regarding the adverse effects of widespread use of antibiotics should be taken into account when choosing a therapeutic strategy of chronic periodontitis. ${ }^{14}$

However, prolonged use of antibiotics can result in the development of resistant strains. With the rise in bacterial resistance to antibiotics, there is considerable interest in the development of other classes of antimicrobials for the control of infection. Hence, we chose a herbal formulation because of its advantages like easy availability, low cost, and less side effects.

However, there are very few in vitro studies attributing to the antibacterial properties of garlic against periodontitis and hardly any clinical trials on effects of garlic on chronic periodontitis.

Hence, the aim of this randomized clinical trial was to evaluate clinical and microbiological outcomes following the application of garlic gel as a LDD in the treatment of chronic periodontitis patients.

Garlic is one of the most extensively investigated medicinal plants since ancient times. With its high trace mineral content and enzymes and sulfur-containing compounds, garlic has shown antiviral, antibacterial, antifungal, anti-inflammatory, and antioxidant abilities. The garlic extract has been shown to have a wide spectrum inhibitory effect on the growth of various grampositive and gram-negative bacteria and is also active against multidrug-resistant organisms such as Pseudomonas aeruginosa, Klebsiella pneumoniae, and Mycobacterium tuberculosis. ${ }^{15}$

Typical volatiles in crushed garlic and garlic essential oil include diallyl sulfide (DAS), diallyl disulfide (DADS), diallyl trisulfide, methyl allyl disulfide, methyl allyl trisulfide, 2-vinyl-1, 3-dithiin, 3-vinyl-1, 2-dithiin, and ajoene. At the same time, glutamylcysteines are converted to $S$-allyl cysteine (SAC) via a pathway other than the alliin/allicin pathway. S-allyl cysteine contributes heavily to the health benefits of garlic.

In the experimental group, the SRP + garlic gel group demonstrated the significant reduction of PI and CAL gain as compared to the control group. The PPD, GI, and GBI demonstrated highly significant reduction in SRP + garlic gel than the SRP group.

The improvement in clinical parameters can be due to antiinflammatory and antioxidant properties of the garlic because of allicin that inhibited the production of pro-inflammatory cytokine messengers in a study of inflammatory bowel disease, apparently by inactivating the pro-inflammatory factor NFkB via its IkB inhibitor. By virtue of sulphur-based antioxidants found in garlic, NFkB was maintained in its inactive state, thus preventing synthesis of excess cyclooxygenase (COX)/lipooxygenase (LOX). ${ }^{16}$

Oxidative stress is recognized as one of the pathogenic mechanisms of chronic inflammatory diseases, including cardiovascular disorders and cancer. Consequently, compounds with antioxidant properties may be used to prevent oxidative stress-mediated diseases. ${ }^{17}$

Abundant research has established garlic and its organosulfur compounds to be strong antioxidants. Garlic protection against oxidant-induced damage can be credited to major compounds in the garlic extract like SAC and S-allyl mercapto-L-cysteine, by displaying the radical scavenging activity and modulating the cellular antioxidant enzyme activity. Antioxidant properties of garlic have been demonstrated in animal disease models. The aqueous garlic extract reduces oxidative stress and prevents vascular remodeling by suppressing $\mathrm{NAD}(\mathrm{P}) \mathrm{H}$-oxidase in the fructose-induced metabolic syndrome model in rats. ${ }^{18} \mathrm{~S}$-Allylcysteine also reduces lipid peroxidation and superoxide radical production, and elevates the Cu-Zn-superoxide dismutase activity in 1-methyl4-phenylpyridinium-induced parkinsonism in mice.

Overall, the garlic gel brought about clinically statistically significant reduction of plaque, gingival inflammation, gingival bleeding, probing depth, and CAL.

\section{Conclusion}

In the present study, use of garlic gel as a local drug in adjunct with SRP has shown good results when compared to SRP alone.

These results conclude that garlic gel can be a used as an antiplaque, antibacterial, and anti-inflammatory agent in adjunct to SRP. However, as previously mentioned, this is the first of its kind study to the best of our knowledge. Further research with larger samples needs to be focused to confirm the use of garlic in clinical practice.

\section{References}

1. Reddy PD, Satyanarayana T, Purushothaman M. Local drug delivery of herbs for treatment of periodontitis. J Inno Tren Pharm Sci 2010;1:245-251.

2. Kornman KS. Controlled release local delivery-antimicrobials in periodontics. Prospects for the future. J Periodontol 1993;64(8 Suppl): 782-791. DOI: 10.1902/jop.1993.64.8s.782.

3. Genco RJ. Host responses in periodontal diseases: current concepts. J Periodontol. 1992 Apr;63(4 Suppl):338-355.

4. Perinetti G, Paolantonio M,Cordella C, etal.Clinical and microbiological effects of subgingival administration of two active gels on persistent pockets of chronic periodontitis patients. J Clin Periodontol 2004;31(4):273-281. DOI: 10.1111/j.1600-051x.2004.00481.x.

5. Paolantonio M, D'Angelo M, Grassi RF, et al. Clinical and microbiologic effects of subgingival controlled-release delivery of chlorhexidine chip in the treatment of periodontitis: a multicenter study. J Periodontol 2008;79(2):271-282. DOI: 10.1902/jop.2008.070308.

6. Pistorius A, Willershausen B, Steinmeier EM, et al. Efficacy of subgingival irrigation using herbal extracts on gingival inflammation. J Periodontol 2003;74(5):616-622. DOI: 10.1902/jop.2003.74.5.616.

7. Chitme HR, Chandra R, Kaushik S. Studies on anti-diarrhoeal activity of calotropis gigantea in experimental animals. J Pharm Pharm Sci 2003;7(1):70-75.

8. Dhinahar S, Lakshmi T. Role of botanicals as antimicrobial agents in management of dental infections-a review. Int J Phar Biosci 2011;2(4):B690-B704.

9. Block E. The chemistry of garlic and onions. Sci Am 1985;252(3): 114-119. DOI: 10.1038/scientificamerican0385-114.

10. Armitage GC. Development of a classification system for periodontal diseases and conditions. Ann Periodontol 1999;4(1):1-6. DOI: 10.1902/ annals.1999.4.1.1.

11. Antcziik-Bouckoms AA, Tulloch JFC, Berkey CS. Split-mouth and cross-over designs in dental research. J Clin Periodontol 1990; 17(7 Pt 1):446-453.

12. Stambaugh RV, Dragoo $M$, Smith DM, et al. The limits of subgingival Scaling. Int J Periodontics Restorative Dent 1981;1(5):30-41.

13. Lindhe J, Heijl L, Goodson JM, et al. Local tetracycline delivery using hollow fiber devices in periodontal therapy. J Clin Periodontol 1979;6(3):141-149. DOI: 10.1111/j.1600-051X.1979.tb02193.x.

14. Pavia M, Nobile CG, Angelillo IF. Meta-analysis of local tetracycline in treating chronic periodontitis. J Periodontol 2003 Jun;74(6):916-932. DOI: 10.1902/jop.2003.74.6.916.

15. Fani MM, Kohanteb J, Dayaghi M. Inhibitory activity of garlic (Allium sativum) extract on multidrug-resistant Streptococcus mutans. J Indian Soc Pedodont Prev Dent 2007;25(4):164-168. DOI: 10.4103/0970-4388.37011. 
16. Lang A, Lahav M, Sakhnini E, et al. Allicin inhibits spontaneous and TNF- $a$ induced secretion of proinflammatory cytokines and chemokines from intestinal epithelial cells. Clin Nutr 2004;23(5): 1199-1208. DOI: 10.1016/j.clnu.2004.03.011.

17. Mayne ST. Antioxidant nutrients and chronic disease: use of biomarkers of exposure and oxidative stress status in epidemiologic research. J Nutr 2003;133(3):933-940. DOI: 10.1093/jn/133 3.933S.

18. Vazquez-Prieto MA, Gonzalez RE, Renna NF, et al. Aqueous garlic extracts prevent oxidative stress and vascular remodeling in an experimental model of metabolic syndrome. J Agric Food Chem 2010;58(11):630-635. DOI: 10.1021/jf1006819. 